\title{
Strip load on transversely isotropic elastic double porosity media with strong permeability contrast
}

\author{
Qi Zhang ${ }^{\mathbb{*} *}$ \\ Department of Civil and Environmental Engineering, Stanford University, Stanford CA 94305, USA
}

\author{
Keywords: \\ Double porosity \\ transverse isotropy \\ non-Darcy flow \\ strip load \\ Cited as: \\ Zhang, Q. Strip load on transversely \\ isotropic elastic double porosity media with \\ strong permeability contrast. Advances in \\ Geo-Energy Research, 2021, 5(4): 353-364, \\ doi: 10.46690/ager.2021.04.02
}

\begin{abstract}
:
In double porosity media, fluid could flow through the distributed micro-fractures at a larger scale as well as through the nanopores of the organic matrix at a smaller scale, while fluid mass could be transferred between them. Besides, the solid deformation acts as an important coupling term and leads to the two-way coupled process. However, these processes are not well presented in previous studies. In this work, a new coupled modeling approach was proposed for saturated double porosity media with strong permeability contrast. Specifically, the transversely isotropic equivalent fracture permeability is considered for the preferentially oriented micro-fractures at the micro-scale or meso-scale; while at the nano-scale, the non-Darcy flow and mass transfer equations are employed to consider the interaction between fluid particles and the solid pore wall. Finally, the stabilized finite element method is developed to investigate the settlement and pressure dissipation behaviors of the strip load problem.
\end{abstract}

\section{Introduction}

Coupled flow and deformation is always an intriguing research topic (Rutqvist and Stephansson, 2003) and is important for industry as well (Cui et al., 2018). The earliest research could date back to Terzaghi and Biot, who established the basis of poroelasticity (Lewis et al., 1998; Ye et al., 2021), which is still a powerful tool in many engineering problems nowadays (Chen and Baker, 2019; Chen et al., 2021). The poroelasticity theory assumes the porous media is homogeneous, which belongs to the category of single porosity poromechanical model (Zhang and Mehrabian, 2020, 2021). However, in the natural environment, the porous media could be highly heterogeneous, which means there might be more than one porosity scale of interest (Kim et al., 2012). Here a special category named double porosity fractured media was considered, which is proposed by researchers in petroleum engineering (Barenblatt et al., 1960; Warren and Root, 1963). Double porosity fractured media has micro-fractures at the micro-scale or meso-scale, and nanoporous matrix at the nano-scale. It should be noted that the macro-fractures and faults belong to another much larger porosity scale (Wang et al., 2020; Yang et al., 2021), which is not covered here.
${ }^{*}$ Corresponding author

E-mail address: qzhang94@stanford.edu (Q. Zhang).
Over the last 50 years, numerous frameworks with different degrees of sophistication have been proposed to model the double porosity fractured media. Here only the dual-continuum conceptual framework is used (Coussy, 2003), which assumes two pore regions overlap with each other in the computational domain, and they are linked by a leakage (source/sink) term (Lemonnier and Bourbiaux, 2010). Based on this framework, many researchers have investigated the coupled flow and deformation behaviors in double porosity fractured media (Bai et al., 1993). However, most of these publications relied on the phenomenological approach to obtain the poromechanical coefficients, or they adopted two separate constitutive laws for two continua.

For the fluid flow behavior, most of the previous studies assumed it would follow Darcy's law (Shao et al., 2020b; Zhang et al., 2020). However, with the development of unconventional reservoirs, more and more attentions have been paid to the flow mechanisms in matrix pores whose size is less than 1 micrometer such as the pores in the organic matrix of shale. Experimental studies showed the fluid flux deviated from what is calculated using Darcy's law, and they believed the interaction between fluid particles and the solid pore wall leads to this deviation. Generally speaking, there are three types of

\section{Scientific}

Press 
non-Darcy flow: high-velocity Forchheimer flow (Shao et al., 2020a), gas slippage with Knudsen diffusion (Li et al., 2020), and low-velocity non-Darcy flow of liquid (Hansbo, 1997; Li et al., 2016). For this work, only the last type was considered. Besides the fluid flux, in double porosity fractured media, the leakage parameter exhibits a similar behavior, which is also caused by the aforementioned interaction. Therefore, without causing any confusion, the term "non-Darcy flow" applies to both the flow through nanoporous matrix and the mass exchange.

Anisotropy is a ubiquitous property of natural geomaterials such as shale (Liao and Yang, 2021; Liao et al., 2021). In other words, the material properties show directional dependence (Zhu et al., 2020). A very special case of anisotropy is transverse isotropy, in which there exists a plane of isotropy defined by its normal vector (Xiao and Yue, 2020). Transverse isotropy could affect both mechanical properties and fluid flow properties (Baker and Chen, 2020; Chen and Baker, 2021; Zhang and Wang, 2021). Therefore, it is necessary to build a comprehensive coupled flow and deformation model encapsulating these essential features.

In this work, we develop a framework for hydromechanical modeling of double porosity media with strong permeability contrast in which fluid flow through the micro-fracture network continuum exhibits transverse isotropy while fluid flow through the porous matrix continuum follows a low-velocity non-Darcy law. The framework is comprised of conservation laws, constitutive laws, and the finite element formulation. Finally, the framework is applied to a practical example to demonstrate the impacts of double porosity, transverse isotropy, and non-Darcy flow.

\section{Conservation laws}

\subsection{Balance of mass}

For double porosity media, the balance of mass equations start from the fundamental/primitive forms (Coussy, 2003):

$$
\begin{gathered}
\frac{\partial \rho^{s}}{\partial t}+\nabla \cdot\left[\rho_{s}(1-\phi) \vec{v}_{s}\right]=0 \\
\frac{\partial\left(\rho_{f 1} \phi_{1}\right)}{\partial t}+\nabla \cdot\left(\rho_{f 1} \phi_{1} \vec{v}_{1}\right)=\rho_{f} \gamma\left(p_{2}-p_{1}\right) \\
\frac{\partial\left(\rho_{f 2} \phi_{2}\right)}{\partial t}+\nabla \cdot\left(\rho_{f 2} \phi_{2} \vec{v}_{2}\right)=\rho_{f} \gamma\left(p_{1}-p_{2}\right)
\end{gathered}
$$

where $\rho^{s}=\rho_{s}(1-\phi)$ is the partial density of the solid material, $\rho_{s}$ is the intrinsic density of the solid material, $\vec{v}_{s}$ is the velocity of the solid skeleton, $\phi=\phi_{1}+\phi_{2}$ is the total porosity, $\phi_{1}$ and $\phi_{2}$ are individual porosities for two continua, $\rho_{f 1}$ and $\rho_{f 2}$ are fluid densities in two continua, $\vec{v}_{1}$ and $\vec{v}_{2}$ are intrinsic flow velocities in two continua, $p_{1}$ and $p_{2}$ are fluid pressures in two continua, $\rho_{f}$ is the reference fluid density, and $\gamma$ is the leakage parameter.

Next, by adopting the material time derivative of the solid motion and barotropic flows, above three equations could be rewritten as:

$$
\frac{1-\phi}{K_{s}} \frac{\mathrm{d} p_{s}}{\mathrm{~d} t}-\frac{\mathrm{d} \phi}{\mathrm{d} t}+(1-\phi) \nabla \cdot \vec{v}_{s}=0
$$

$\frac{\phi_{1}}{K_{f 1}} \frac{\mathrm{d} p_{1}}{\mathrm{~d} t}+\frac{\mathrm{d} \phi_{1}}{\mathrm{~d} t}+\phi_{1} \nabla \cdot \vec{v}_{s}+\frac{1}{\rho_{f 1}} \nabla \cdot\left(\rho_{f 1} \vec{q}_{1}\right)=\left(\frac{\rho_{f}}{\rho_{f 1}}\right) \gamma\left(p_{2}-p_{1}\right)$

$$
\frac{\phi_{2}}{K_{f 2}} \frac{\mathrm{d} p_{2}}{\mathrm{~d} t}+\frac{\mathrm{d} \phi_{2}}{\mathrm{~d} t}+\phi_{2} \nabla \cdot \vec{v}_{s}+\frac{1}{\rho_{f 2}} \nabla \cdot\left(\rho_{f 2} \vec{q}_{2}\right)=\left(\frac{\rho_{f}}{\rho_{f 2}}\right) \gamma\left(p_{1}-p_{2}\right)
$$

where $K_{S}$ is the bulk modulus of the solid material, $p_{s}$ is the solid pressure, $K_{f 1}$ and $K_{f 2}$ are fluid compressibilities, $\vec{q}_{1}$ and $\vec{q}_{2}$ are superficial Darcy velocities defined as $\vec{q}_{1}=\phi_{1}\left(\vec{v}_{1}-\vec{v}_{s}\right)$ and $\vec{q}_{2}=\phi_{2}\left(\vec{v}_{2}-\vec{v}_{s}\right)$.

In order to further simplify Eq. (4), a function $\Gamma$ was proposed such that it satisfies:

$$
\Gamma\left(p_{s}, \phi, p\right)=0
$$

where $p=\chi p_{1}+(1-\chi) p_{2}$ represents a weighted average fluid pressure $(0 \leq \chi \leq 1)$. By taking time derivative on both sides of Eq. (7):

$$
\frac{\partial \Gamma}{\partial p_{s}} \frac{\mathrm{d} p_{s}}{\mathrm{~d} t}+\frac{\partial \Gamma}{\partial \phi} \frac{\mathrm{d} \phi}{\mathrm{d} t}+\frac{\partial \Gamma}{\partial p} \frac{\mathrm{d} p}{\mathrm{~d} t}=0
$$

By combining Eq. (4) with Eq. (8):

$$
\left(1+\frac{1-\phi}{K_{s}} \frac{\partial \Gamma / \partial \phi}{\partial \Gamma / \partial p_{s}}\right) \frac{\mathrm{d} \phi}{\mathrm{d} t}=(1-\phi) \nabla \cdot \vec{v}_{s}-\frac{1-\phi}{K_{s}} \frac{\partial \Gamma / \partial p}{\partial \Gamma / \partial p_{s}} \frac{\mathrm{d} p}{\mathrm{~d} t}
$$

Suppose that a $\Gamma$ exists such that it satisfies following two equations:

$$
\frac{\partial \Gamma}{\partial p}+\frac{\partial \Gamma}{\partial p_{s}}=0
$$

$$
\frac{(1-\phi)^{2} K_{s} \mathcal{A}}{K_{s}+(1-\phi) \mathcal{A}}=K
$$

where $K$ is the drained bulk modulus and

$$
\mathcal{A}=\frac{\partial \Gamma / \partial \phi}{\partial \Gamma / \partial p_{s}}
$$

Eq. (9) can be simplified into:

$$
\frac{\mathrm{d} \phi}{\mathrm{d} t}=\left(1-\frac{K}{K_{s}}-\phi\right) \nabla \cdot \vec{v}_{s}+\frac{1}{K_{s}}\left(1-\frac{K}{K_{s}}-\phi\right) \frac{\mathrm{d} p}{\mathrm{~d} t}
$$

The familiar Biot coefficient can be identified as (Cheng, 2016; Shao et al., 2021):

$$
\alpha=1-\frac{K}{K_{s}}
$$

In the special case of $K_{f 1}=K_{f 2}=K_{f}$ and $p_{1}=p_{2}=p$, Eqs. (5), (6), and (13) can be combined to obtain the so-called Biot modulus $M$ (Cheng, 2016) in this novel way:

$$
\frac{1}{M}=\frac{\phi}{K_{f}}+\frac{\alpha-\phi}{K_{s}}
$$


Now the question is whether this $\Gamma$ exists? The answer is yes and you can verify the following $\Gamma$ satisfies Eqs. (10) and (11):

$$
\begin{aligned}
\Gamma= & K_{s} \ln \left(\frac{1-\phi}{1-\frac{K}{K_{s}}-\phi}\right)+p_{s}-p \\
& -K_{s} \ln \left(\frac{1-\phi_{0}}{1-\frac{K}{K_{s}}-\phi_{0}}\right)-p_{s}^{0}+p^{0}
\end{aligned}
$$

The last three terms on the right-hand side make $\Gamma=0$ at $t=0$. When $K_{s} \rightarrow+\infty$, the limit of $\Gamma$ is given as:

$$
\lim _{K_{s} \rightarrow+\infty} \Gamma=\frac{K}{1-\phi}+p_{s}-p-\frac{K}{1-\phi_{0}}-p_{s}^{0}+p^{0}
$$

In the following context, several stronger assumptions are made such that $K_{s}=K_{f 1}=K_{f 2}=+\infty, \rho_{f 1}=\rho_{f 2}=\rho_{f}$, and the internal structure of the double porosity media is not evolved (Zhang, 2020) due to a lack of the experimental evidence, i.e., $\phi_{1} /\left(\phi_{1}+\phi_{2}\right)$ is a constant. Under these assumptions, Eqs. (5) and (6) can be rewritten as:

$$
\begin{aligned}
& \alpha_{1} \nabla \cdot \vec{v}_{s}+\nabla \cdot \vec{q}_{1}=\gamma\left(p_{2}-p_{1}\right) \\
& \alpha_{2} \nabla \cdot \vec{v}_{s}+\nabla \cdot \vec{q}_{2}=\gamma\left(p_{1}-p_{2}\right)
\end{aligned}
$$

where $\alpha_{1}=\phi_{1} /\left(\phi_{1}+\phi_{2}\right)$ and $\alpha_{2}=1-\alpha_{1}$ are known as double porosity Biot coefficients.

\subsection{Balance of linear momentum}

The balance of linear momentum is formulated with respect to the bulk material and it is given as:

$$
\nabla \cdot \sigma+\rho \vec{g}=\overrightarrow{0}
$$

where $\sigma$ is the total stress tensor of the bulk material, $\rho=\rho_{s}\left(1-\phi_{1}-\phi_{2}\right)+\rho_{f}\left(\phi_{1}+\phi_{2}\right)$ is the bulk density, and $\vec{g}$ is the gravity acceleration vector. The total stress tensor $\sigma$ contains contributions from fluid pressures $p_{1}$ and $p_{2}$, and we use the following effective stress principle to decompose $\sigma$ :

$$
\boldsymbol{\sigma}^{\prime}=\boldsymbol{\sigma}+\alpha_{1} p_{1} \mathbf{1}+\alpha_{2} p_{2} \mathbf{1}
$$

where $\sigma^{\prime}$ is known as the effective stress tensor and it purely depends on the deformation field, $\mathbf{1}$ is the second-order identity tensor.

\section{Constitutive laws}

To close the boundary value problem, constitutive relations are needed. The number of required constitutive relations could be identified from the thermodynamics of porous media (Coussy, 2003).

Based on the theory of linear elasticity, $\sigma^{\prime}$ is linearly related to the strain tensor $\epsilon$ as (Villamor Lora et al., 2016) (assuming 3D, thus $z$ axis corresponds to the vertical direction, and a slight modification is needed for the $2 \mathrm{D}$ case in which $y$ axis corresponds to the vertical direction):

$$
\left\{\begin{array}{c}
\sigma_{x}^{\prime} \\
\sigma_{y}^{\prime} \\
\sigma_{z}^{\prime} \\
\tau_{x y}^{\prime} \\
\tau_{x z}^{\prime} \\
\tau_{y z}^{\prime}
\end{array}\right\}=\left[\begin{array}{cccccc}
\frac{1}{E_{h}} & -\frac{v_{h h}}{E_{h}} & -\frac{v_{v h}}{E_{v}} & & & \\
-\frac{v_{h h}}{E_{h}} & \frac{1}{E_{h}} & -\frac{v_{v h}}{E_{v}} & & & \\
-\frac{v_{h y}}{E_{h}} & -\frac{v_{h v}}{E_{h}} & \frac{1}{E_{v}} & & & \\
& & & \frac{2\left(1+v_{h h}\right)}{E_{h}} & & \\
& & & & \frac{1}{G_{v h}} & \\
& & & & & \frac{1}{G_{v h}}
\end{array}\right]^{-1}\left\{\begin{array}{c}
\varepsilon_{x} \\
\varepsilon_{y} \\
\varepsilon_{z} \\
2 \varepsilon_{x y} \\
2 \varepsilon_{x z} \\
2 \varepsilon_{y z}
\end{array}\right\}
$$

where all the constitutive parameters $E_{v}, E_{h}, G_{v h}, v_{h h}, v_{h v}$, and $v_{v h}$ are measurable in lab experiments (note $v_{v h} / E_{v}=v_{h v} / E_{h}$ ), and their physical meanings are given in Villamor Lora et al. (2016). Above equation can be briefly written as $\left\{\sigma^{\prime}\right\}=C^{e}\{\varepsilon\}$ where $C^{e}$ is known as the $6 \times 6$ stiffness matrix and it is the Voigt form of the fourth-order elastic tensor $\mathbb{C}^{e}$.

The flow in the micro-fracture network continuum follows Darcy's law, which is given by the linear relation:

$$
\vec{q}_{2}=-\frac{\boldsymbol{k}_{2}}{\mu_{f}} \cdot\left(\nabla p_{2}-\rho_{f} \vec{g}\right)
$$

where $\boldsymbol{k}_{2}$ is the equivalent fracture permeability (Zhang et al., 2021) that takes the form:

$$
k_{2}=k_{\min } \vec{n}_{2} \otimes \vec{n}_{2}+k_{\max }\left(\mathbf{1}-\vec{n}_{2} \otimes \vec{n}_{2}\right)
$$

This mathematical expression characterizes a transversely isotropic material with respect to fluid flow.

As for the porous matrix continuum, $\vec{q}_{1}$ follows the lowvelocity non-Darcy flow of liquid that can be expressed as a nonlinear function of $\vec{\varphi}=\nabla p_{1}-\rho_{f} \vec{g}$. Here we extend the scalar equation proposed by Hansbo (1997) to the vectorial form in 3D together with a no flow part. The equation is given as follows:

$$
\left(\vec{q}_{1}\right)_{j}= \begin{cases}0 & \left|\varphi_{j}\right|<\lambda_{\min } \\ -\frac{k_{1}\left(\left|\varphi_{j}\right|-\lambda_{\min }\right)^{\xi}}{\mu_{f} \xi \delta^{\xi-1}} \operatorname{sgn}\left(\varphi_{j}\right) & \lambda_{\min } \leq\left|\varphi_{j}\right| \leq \lambda_{\max } \\ -\frac{k_{1}}{\mu_{f}}\left(\left|\varphi_{j}\right|-\lambda_{\min }-\frac{\xi-1}{\xi} \delta\right) \operatorname{sgn}\left(\varphi_{j}\right) & \left|\varphi_{j}\right|>\lambda_{\max }\end{cases}
$$

where the physical meanings of all parameters can be found in Zhang et al. (2021). Later in the finite element formulation, the construction of Jacobian matrix requires the expression for $\partial \vec{q}_{1} / \partial \vec{\varphi}$, which is a $n_{\operatorname{dim}} \times n_{\operatorname{dim}}$ diagonal matrix, and the diagonal element is given as $\left(j=1, \cdots, n_{\mathrm{dim}}\right)$ :

$$
\left(\frac{\partial \vec{q}_{1}}{\partial \vec{\varphi}}\right)_{j j}=\frac{\partial\left(\vec{q}_{1}\right)_{j}}{\partial \varphi_{j}}= \begin{cases}0 & \left|\varphi_{j}\right|<\lambda_{\min } \\ -\frac{k_{1}\left(\left|\varphi_{j}\right|-\lambda_{\min }\right)^{\xi-1}}{\mu_{f} \delta^{\xi-1}} & \lambda_{\min } \leq\left|\varphi_{j}\right| \leq \lambda_{\max } \\ -\frac{k_{1}}{\mu_{f}} & \left|\varphi_{j}\right|>\lambda_{\max }\end{cases}
$$

While the flux of fluid through the porous matrix continuum may be lower than that estimated from the Darcy's law due to boundary layer effects, the leakage parameter $\gamma$ could also change with $\vec{\varphi}$. From Khalili et al. (1999), $\gamma$ is closely related to the apparent permeability of the matrix continuum given in Eq. (26). Therefore, $\gamma$ should be a piecewise function with three stages, separated by $\lambda_{\min }$ and $\lambda_{\max }$. Under the 
situations of Darcy flow $\left(x>\lambda_{\max }\right)$ and no-flow $\left(x<\lambda_{\min }\right)$, $\gamma$ should be a constant. In the middle transition stage, the cosine function could make the first-order derivative $\gamma^{\prime}$ still continuous at $\lambda_{\min }$ and $\lambda_{\max }$. The exponent parameter $\xi$ is also included in the cosine term. The final form of $\gamma$ is given as:

$$
\gamma= \begin{cases}\gamma_{\min } & \varphi_{\max }<\lambda_{\min } \\ \frac{\gamma_{\max }+\gamma_{\min }}{2}+\frac{\gamma_{\min }-\gamma_{\max }}{2} \cos \left[\pi\left(\frac{\varphi_{\max }-\lambda_{\min }}{\delta}\right)^{\xi}\right] & \lambda_{\min } \leq \varphi_{\max } \leq \lambda_{\max } \\ \gamma_{\max } & \varphi_{\max }>\lambda_{\max }\end{cases}
$$

where $\gamma_{\max }$ and $\gamma_{\min }$ are the maximum and minimum leakage parameters, respectively, and

$$
\varphi_{\max }=\max _{j \in\left\{1, \cdots, n_{\mathrm{dim}}\right\}}\left|\varphi_{j}\right|
$$

Note that when $\gamma_{\max }=\gamma_{\min }$, the leakage parameter $\gamma$ becomes a trivial constant as in the Warren and Root model (Warren and Root, 1963). A sketch of $\vec{q}_{1}$ and $\gamma$ is given in Fig. 1, which clearly shows differences between Darcy and non-Darcy types of flow behaviors.

In the following numerical simulation, the values of $\lambda_{\min }$, $\lambda_{\max }$, and $\xi$ are user-defined (assumed), while the leakage parameter $\gamma$ is estimated based on the empirical formula (Sarma and Aziz, 2006):

$$
\gamma \approx \frac{\sigma_{\mathrm{sh}}}{l_{c}^{2}} \frac{k_{1}}{\mu_{f}}
$$

where $\sigma_{\mathrm{sh}}$ is known as the shape factor constant (Sarma and Aziz, 2006; Ranjbar and Hassanzadeh, 2011) that is dimensionless, $l_{c}$ is the characteristic length of the matrix block (cut by micro-fracture network) that should be much smaller than the domain size. Here $\sigma_{\mathrm{sh}}$ takes values suggested by Kazemi et al. (1976): $\sigma_{\mathrm{sh}}=8$ for $2 \mathrm{D}$ and $\sigma_{\mathrm{sh}}=12$ for 3D.

\section{Finite element formulation}

The Galerkin method is employed to develop the finite element (FEM) formulation of the coupled flow and deformation problem presented earlier. Here all the symbols are written in the normal font. Without loss of generality, the formulation is in 3D. In FEM, $u \in \mathbb{R}^{3}=N_{u} d, p_{1} \in \mathbb{R}=N_{p} r_{1}$, and $p_{2} \in \mathbb{R}=N_{p} r_{2}$, where $N_{u} \in \mathbb{R}^{3 \times n}$ is the shape function matrix for the displacement field, $d \in \mathbb{R}^{n}$ is the nodal displacement vector, $N_{p} \in \mathbb{R}^{1 \times m}$ is the shape function matrix for both fluid pressures $p_{1}$ and $p_{2}, r_{1} \in \mathbb{R}^{m}$ and $r_{2} \in \mathbb{R}^{m}$ are the nodal fluid pressure vectors. Here $d, r_{1}$, and $r_{2}$ are to be determined. After spatial discretization and time integration, $(n+2 m)$ fully coupled algebraic residual equations were obtained:

$$
\begin{gathered}
\mathcal{R}_{u}=\int_{\Omega} B^{T}\left(\sigma^{\prime}-\alpha_{1} p_{1} \tilde{1}-\alpha_{2} p_{2} \tilde{1}\right) \mathrm{d} V \\
-\int_{\Omega} N_{u}^{T} \rho g \mathrm{~d} V+F_{B . C .} \\
\mathcal{R}_{1}=\int_{\Omega} \alpha_{1} N_{p}^{T} \tilde{1}^{T}\left(\varepsilon-\varepsilon^{[\omega]}\right) \mathrm{d} V-\Delta t \int_{\Omega} E^{T} q_{1} \mathrm{~d} V \\
-\Delta t \int_{\Omega} N_{p}^{T} \gamma^{[\omega]}\left(p_{2}-p_{1}\right) \mathrm{d} V+G_{B . C .}
\end{gathered}
$$
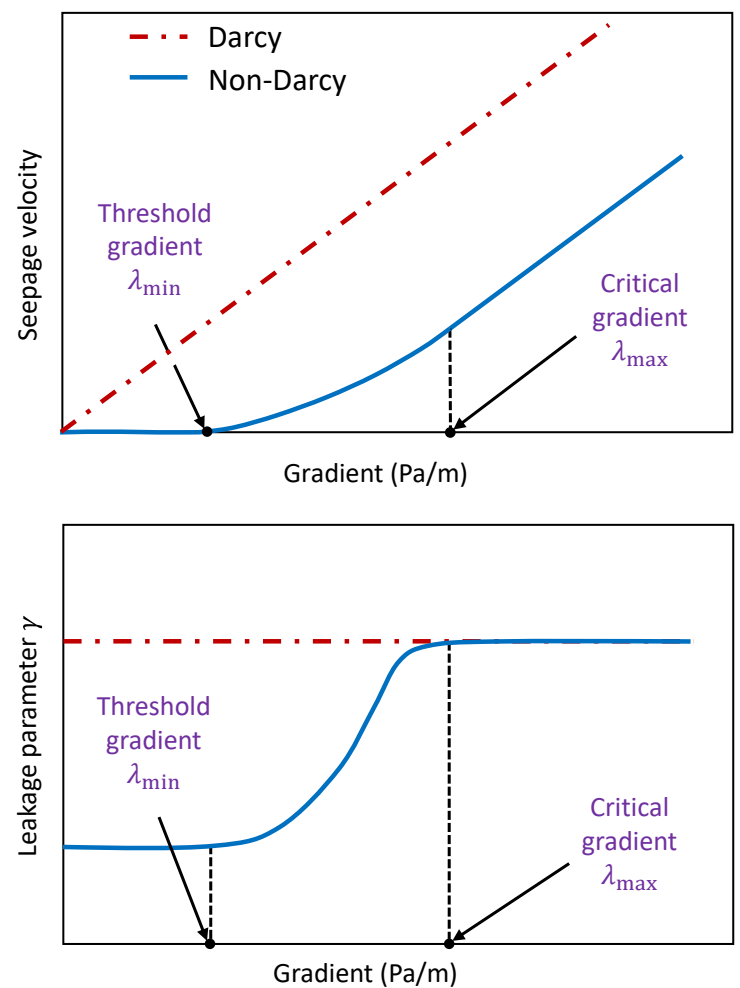

Fig. 1. Type curves of non-Darcy flow and leakage parameter (Zhao et al., 2020). The scale qualification for these nonlinear behaviors would be the same as that for the non-Darcy flow of shale gas.

$$
\begin{aligned}
\mathcal{R}_{2}= & \int_{\Omega} \alpha_{2} N_{p}^{T} \tilde{1}^{T}\left(\varepsilon-\varepsilon^{[\omega]}\right) \mathrm{d} V-\Delta t \int_{\Omega} E^{T} q_{2} \mathrm{~d} V \\
& -\Delta t \int_{\Omega} N_{p}^{T} \gamma^{[\omega]}\left(p_{1}-p_{2}\right) \mathrm{d} V+H_{B . C .}
\end{aligned}
$$

where $B \in \mathbb{R}^{6 \times n}$ is the strain-displacement matrix because the strain "vector" $\varepsilon=B d$ where $\varepsilon=\left[\varepsilon_{x x}, \varepsilon_{y y}, \varepsilon_{z z}\right.$, $\left.2 \varepsilon_{x y}, 2 \varepsilon_{x z}, 2 \varepsilon_{y z}\right]^{T} \in \mathbb{R}^{6}, \sigma^{\prime}=\left[\sigma_{x x}^{\prime}, \sigma_{y y}^{\prime}, \sigma_{z z}^{\prime}, \sigma_{x y}^{\prime}, \sigma_{x z}^{\prime}, \sigma_{y z}^{\prime}\right]^{T} \in \mathbb{R}^{6}$ is the effective stress "vector", $\tilde{1}=[1,1,1,0,0,0]^{T}$ is the Voigt form of the second-order identity tensor, $g \in \mathbb{R}^{3}$ is the gravity acceleration vector, $F_{B . C .} \in \mathbb{R}^{n \times 1}$ represents the contributions from the mechanical traction boundary conditions, $\varepsilon^{[\omega]}$ is the strain "vector" at the previous time step. Here the superscript for quantities at the current time step and iteration counter was omitted. $\Delta t=t^{[\omega+1]}-t^{[\omega]}$ is the time step size, $E \in \mathbb{R}^{3 \times m}=\nabla N_{p}$ leads to $\nabla p_{1}=E r_{1}$ and $\nabla p_{2}=E r_{2}, q_{1} \in \mathbb{R}^{3}$ and $q_{2} \in \mathbb{R}^{3}$ are the superficial velocity vectors, $\gamma^{[\omega]}$ is the leakage parameter at the previous time step, $G_{\text {B.C. }} \in \mathbb{R}^{m \times 1}$ and $H_{B . C .} \in \mathbb{R}^{m \times 1}$ represent the contributions from the flux boundary conditions. Now the tangent operator (or Jacobian, for short) $\mathcal{K} \in \mathbb{R}^{(n+2 m) \times(n+2 m)}$ is:

$$
\underbrace{\left[\begin{array}{ccc}
\mathcal{A} & \mathcal{B} & \mathcal{C} \\
-\mathcal{B}^{T} & \mathcal{D} & \mathcal{E} \\
-\mathcal{C}^{T} & \mathcal{E} & \mathcal{F}
\end{array}\right]}_{\mathcal{K}}\left[\begin{array}{l}
\delta d \\
\delta r_{1} \\
\delta r_{2}
\end{array}\right]=\left[\begin{array}{l}
-\mathcal{R}_{u} \\
-\mathcal{R}_{1} \\
-\mathcal{R}_{2}
\end{array}\right]
$$

The block matrices $\mathcal{A}$ to $\mathcal{F}$ are given as:

$$
\mathcal{A}=\int_{\Omega} B^{T} C^{e} B \mathrm{~d} V
$$




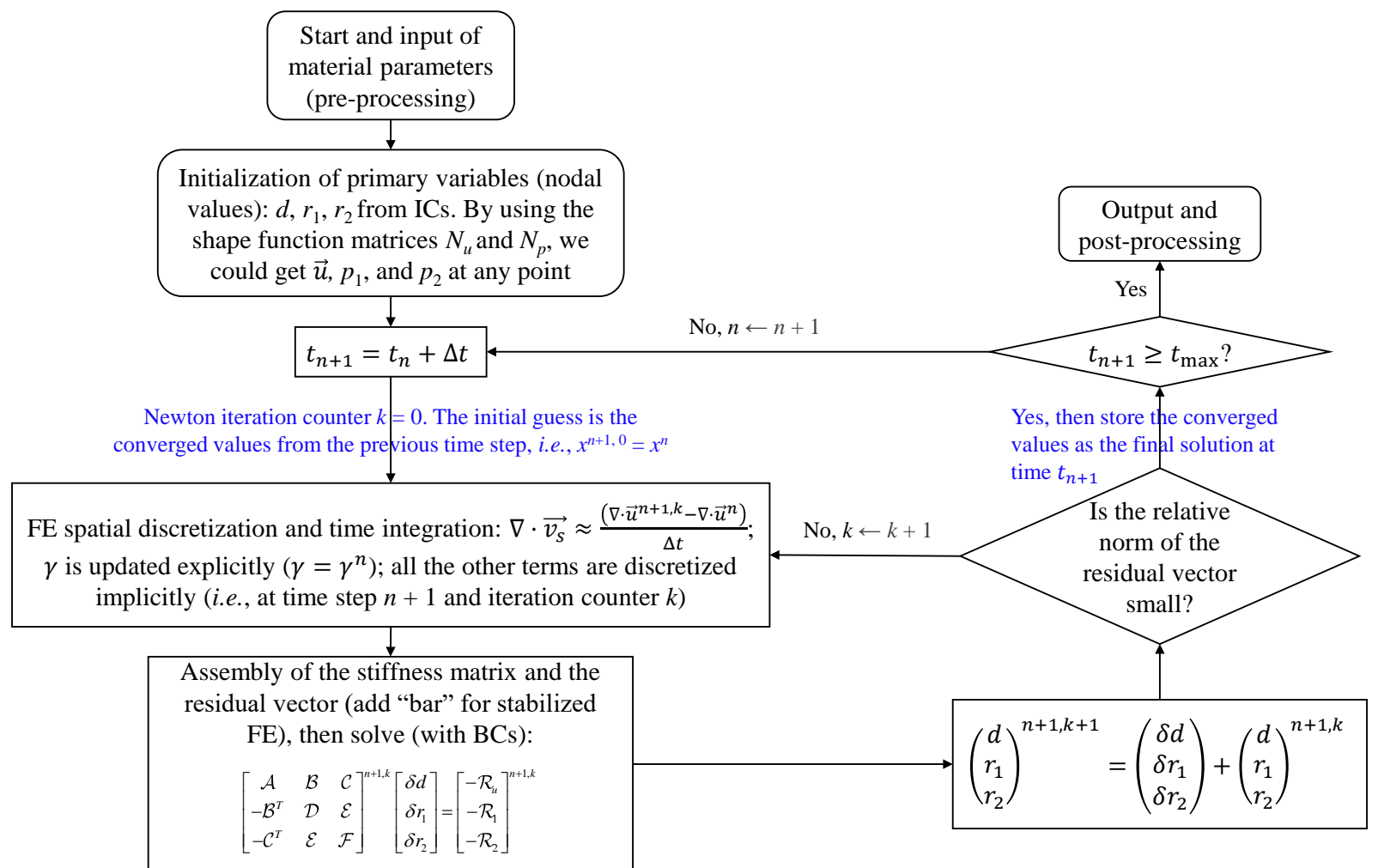

Fig. 2. Schematic of the standard time-dependent FEM implementation. In this flowchart, ICs stand for the initial conditions and BCs stand for the boundary conditions.

$$
\begin{gathered}
\mathcal{B}=-\int_{\Omega} \alpha_{1} B^{T} \tilde{1} N_{p} \mathrm{~d} V \\
\mathcal{C}=-\int_{\Omega} \alpha_{2} B^{T} \tilde{1} N_{p} \mathrm{~d} V \\
\mathcal{D}=-\Delta t \int_{\Omega} E^{T} \frac{\partial \vec{q}_{1}}{\partial \vec{\varphi}} E \mathrm{~d} V+\Delta t \int_{\Omega} N_{p}^{T} \gamma^{[\omega]} N_{p} \mathrm{~d} V \\
\mathcal{E}=-\Delta t \int_{\Omega} N_{p}^{T} \gamma^{[\omega]} N_{p} \mathrm{~d} V \\
\mathcal{F}=\Delta t \int_{\Omega} E^{T} \frac{k_{2}}{\mu_{f}} E \mathrm{~d} V+\Delta t \int_{\Omega} N_{p}^{T} \gamma^{[\omega]} N_{p} \mathrm{~d} V
\end{gathered}
$$

where $k_{2} \in \mathbb{R}^{3 \times 3}$ is $\boldsymbol{k}_{2}$ written in $3 \times 3$ matrix form, $-\partial \vec{q}_{1} / \partial \vec{\varphi} \in \mathbb{R}^{3 \times 3}$ is known as the tangential mobility of the porous matrix continuum whose expression is already given in Eq. (26).

If we adopt equal-order linear interpolations of the displacement field and two pressure fields, the stabilization scheme should be considered. The stabilized residual equations $\overline{\mathcal{R}}_{1}$ and $\overline{\mathcal{R}}_{2}$ are:

$$
\overline{\mathcal{R}}_{1}=\mathcal{R}_{1}+\int_{\Omega} \frac{\tau \alpha_{1}}{2 \bar{G}}\left[N_{p}^{T}-\Pi\left(N_{p}^{T}\right)\right]\left[\bar{p}-\bar{p}^{[\omega]}-\Pi\left(\bar{p}-\bar{p}^{[\omega]}\right)\right] \mathrm{d} V
$$

$$
\begin{gathered}
\overline{\mathcal{R}}_{2}=\mathcal{R}_{2}+\int_{\Omega} \frac{\tau \alpha_{2}}{2 \bar{G}}\left[N_{p}^{T}-\Pi\left(N_{p}^{T}\right)\right]\left[\bar{p}-\bar{p}^{[\omega]}-\Pi\left(\bar{p}-\bar{p}^{[\omega]}\right)\right] \mathrm{d} V \\
\bar{G}=\frac{1}{3}\left[\frac{E_{h}}{2\left(1+v_{h h}\right)}+2 G_{v h}\right]
\end{gathered}
$$

where $\tau$ is the stabilization constant (on the order of 1) that is used to "tune" the level of stabilization (Yan et al., 2020, $2021)^{1}, \bar{G}$ is the generalized shear modulus, $\bar{p}=\alpha_{1} p_{1}+\alpha_{2} p_{2}$ is the weighted pressure (a scalar), $\bar{p}^{[\omega]}$ is $\bar{p}$ at the previous time step, and the projection operator $\Pi(\cdot)$ is defined as:

$$
\Pi(\cdot)=\frac{1}{V_{e}} \int_{\Omega_{e}}(\cdot) \mathrm{d} V
$$

where $V_{e}$ and $\Omega_{e}$ denote the volume and domain of an element, respectively. That's to say, this operator projects a given field to its average value within the element. Due to $\overline{\mathcal{R}}_{1}$ and $\overline{\mathcal{R}}_{2}$, the stabilization of the Jacobian $\mathcal{K}$ was also needed:

$$
\begin{aligned}
& \overline{\mathcal{D}}=\mathcal{D}+\int_{\Omega} \frac{\tau \alpha_{1}^{2}}{2 \bar{G}}\left[N_{p}^{T}-\Pi\left(N_{p}^{T}\right)\right]\left[N_{p}-\Pi\left(N_{p}\right)\right] \mathrm{d} V \\
& \overline{\mathcal{E}}=\mathcal{E}+\int_{\Omega} \frac{\tau \alpha_{1} \alpha_{2}}{2 \bar{G}}\left[N_{p}^{T}-\Pi\left(N_{p}^{T}\right)\right]\left[N_{p}-\Pi\left(N_{p}\right)\right] \mathrm{d} V \\
& \overline{\mathcal{F}}=\mathcal{F}+\int_{\Omega} \frac{\tau \alpha_{2}^{2}}{2 \bar{G}}\left[N_{p}^{T}-\Pi\left(N_{p}^{T}\right)\right]\left[N_{p}-\Pi\left(N_{p}\right)\right] \mathrm{d} V
\end{aligned}
$$

\footnotetext{
${ }^{1}$ A single $\tau$ would be enough for both $\overline{\mathcal{R}}_{1}$ and $\overline{\mathcal{R}}_{2}$ because the weak inf-sup condition is imposed on $\bar{p}$.
} 


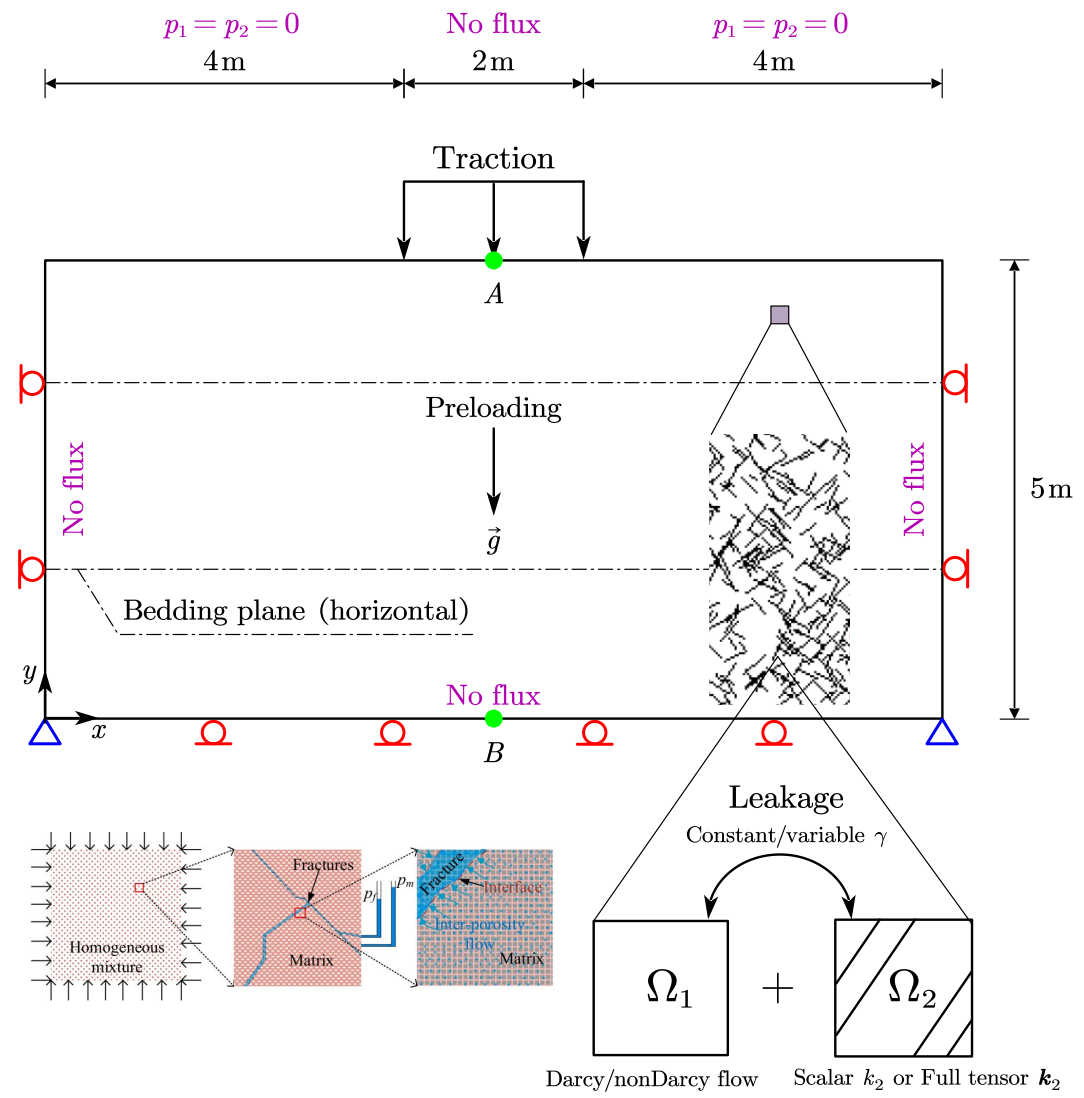

Fig. 3. Schematic of the plane strain strip load problem: the geometry, boundary, and loading conditions. Gravity is considered in this example. Variation in $\rho$ due to changes of porosities is assumed to be very small. The origin $(0,0)$ coincides with the lower-left corner of the domain.

Consequently, the modified tangent operator $\overline{\mathcal{K}}$ satisfies:

$$
\underbrace{\left[\begin{array}{ccc}
\mathcal{A} & \mathcal{B} & \mathcal{C} \\
-\mathcal{B}^{T} & \overline{\mathcal{D}} & \overline{\mathcal{E}} \\
-\mathcal{C}^{T} & \overline{\mathcal{E}} & \overline{\mathcal{F}}
\end{array}\right]}_{\overline{\mathcal{K}}}\left[\begin{array}{l}
\delta d \\
\delta r_{1} \\
\delta r_{2}
\end{array}\right]=\left[\begin{array}{l}
-\mathcal{R}_{u} \\
-\overline{\mathcal{R}}_{1} \\
-\overline{\mathcal{R}}_{2}
\end{array}\right]
$$

A complete and brief FEM implementation is summarized in Fig. 2.

\section{Strip load on a double porosity medium}

\subsection{Problem description}

A 2D rectangular double porosity medium $10 \mathrm{~m}$ wide and $5 \mathrm{~m}$ deep is shown in Fig. 3. Here a full mesh instead of a half mesh was used to define the computational domain because $\boldsymbol{k}_{2}$ may not be isotropic. For the problem initialization, a preloading stage generates steady state fluid pressures $p_{1}=$ $p_{2}=\rho_{f} g(H-y)$ and initial preloading settlement induced by gravity, where $H$ is the depth (here is $5 \mathrm{~m}$ ). Then we apply the strip load $p_{0}=150 \mathrm{kPa}$ instantaneously, which leads to excess pressures. As time goes on, the excess parts will finally vanish.

This problem is a simple schematization of the shallow foundation of a structure, using a long strip foundation made of concrete, for instance. In the case of a permanent load applied to this shallow foundation, it can be expected that pore pressures will be developed below the foundation, and these pore pressures will dissipate in course of time due to the fact that pore water is expelled from the soil. In some clays, this may take a certain time, due to the small permeability. This process is called consolidation. In our situation, there are both horizontal flow and vertical flow, so it is a $2 \mathrm{D}$ consolidation problem. Furthermore, the "foundation soil" contains microfractures. It will be interesting to explore the consolidation patterns under these special settings.

The boundary conditions are highlighted here. For the bottom boundary $(y=0), u_{y}=0, \tau_{x y}=0$, and $q_{1 y}=q_{2 y}=0$. For the two lateral boundaries $(x=0$ or $x=10 \mathrm{~m}), u_{x}=0$, $\tau_{x y}=0$, and $q_{1 x}=q_{2 x}=0$. For the top boundary $(y=5 \mathrm{~m}$ and the unit of $x$ is $\mathrm{m}$ ):

$$
\left.\begin{array}{ll}
p_{1}=p_{2}=0, \sigma_{y y}=\tau_{x y}=0, & 0 \leq x \leq 4 \\
q_{1 y}=q_{2 y}=0, \tau_{x y}=0, \sigma_{y y}=-p_{0}, & 4<x<6 \\
p_{1}=p_{2}=0, \sigma_{y y}=\tau_{x y}=0, & 6 \leq x \leq 10
\end{array}\right\}
$$

\subsection{Simulation parameters}

Three cases are analyzed here. We give the entire parameter set for the first case and mention the differences for the remaining two cases from the first case. The first case can be regarded as the "basic" case: the permeability for the micro-fracture network continuum $\boldsymbol{k}_{2}$ is isotropic and the flow through the porous matrix follows Darcy's law $(\xi=1$, $\lambda_{\min }=0$ ). The parameters are given as: fluid density $\rho_{f}=1000$ 


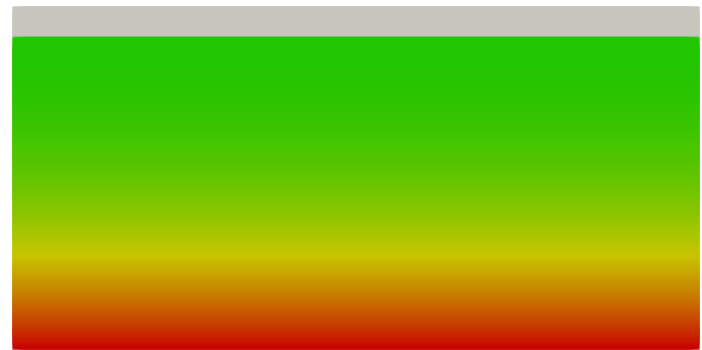

(a)

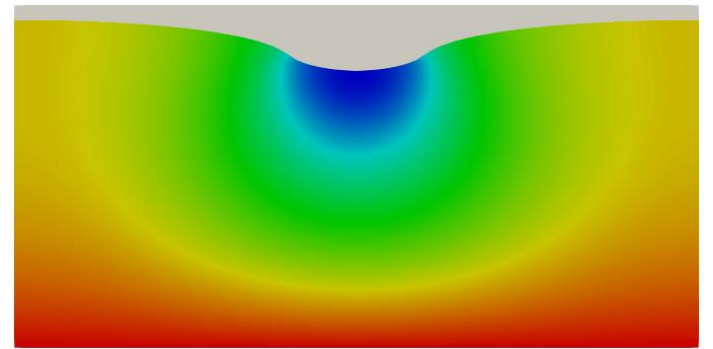

(b)

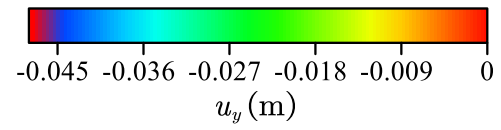

Fig. 4. (a) Preloading deformation on the deformed mesh (magnification of 20) due to gravity. (b) Immediate undrained deformation (including preloading deformation) on the deformed mesh (magnification of 20) due to strip load. The color bar is from $-0.048 \mathrm{~m}$ to 0 .

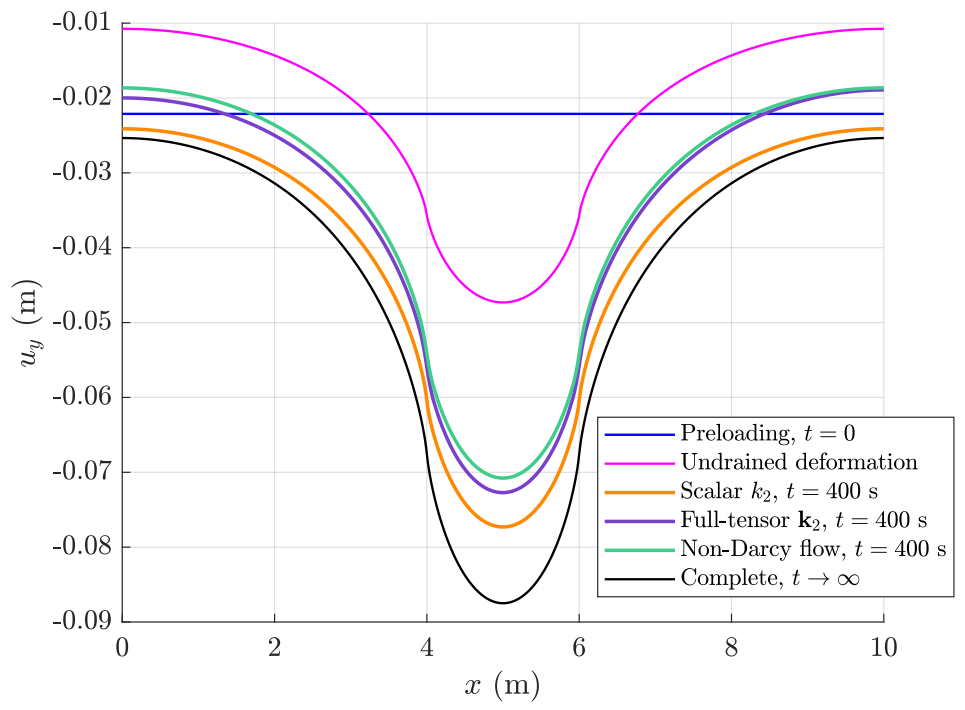

Fig. 5. Ground surface displacement $u_{y}$ (including preloading displacement).

$\mathrm{kg} / \mathrm{m}^{3}$, fluid viscosity $\mu_{f}=0.001 \mathrm{~Pa} \cdot \mathrm{s}, k_{\max }=k_{\min }=10^{-10}$ $\mathrm{m}^{2}, k_{1}=5 \times 10^{-16} \mathrm{~m}^{2}, \gamma_{\max }=\gamma_{\min }=0.24 \mathrm{GPa}^{-1} \cdot \mathrm{s}^{-1}\left(l_{c} \approx\right.$ $0.129 \mathrm{~m})$, initial $\phi_{1}$ is $\phi_{1}^{0}=0.15$, initial $\phi_{2}$ is $\phi_{2}^{0}=0.1$, $\alpha_{1}=0.6, \alpha_{2}=0.4, \rho_{s}=2600 \mathrm{~kg} / \mathrm{m}^{3}, E_{h}=6670.59 \mathrm{kPa}$, $E_{v}=6300 \mathrm{kPa}, v_{h h}=0.1, v_{v h}=0.15$, and $G_{v h}=2750 \mathrm{kPa}$. The second case only makes $\boldsymbol{k}_{2}$ to be transversely isotropic by assigning $\vec{n}_{2}=[-\sin \theta, \cos \theta]^{T}, \theta=\pi / 18, k_{\max }=10^{-10}$ $\mathrm{m}^{2}$, and $k_{\min }=5 \times 10^{-12} \mathrm{~m}^{2}$. The third case assumes $\xi=1.3$, $\lambda_{\max }=19.62 \mathrm{kPa} / \mathrm{m}, \lambda_{\min }=0, \gamma_{\max }=0.24 \mathrm{GPa}^{-1} \cdot \mathrm{s}^{-1}$, and $\gamma_{\min }=0.1 \gamma_{\max }$, while $k_{2}$ is still isotropic as in the first case.

The constrained modulus $D_{v}$ in the vertical direction for this transversely isotropic material is defined as:

$$
\frac{1}{D_{v}}=\frac{1}{E_{v}}-\frac{2\left(v_{v h} / E_{v}\right)^{2} E_{h}}{1-v_{h h}}
$$

We can then calculate the surface displacement $u_{y}$ in the preloading stage by using this constrained modulus, and the result is known as $u_{\text {pre }}$ :

$$
u_{\mathrm{pre}}=\frac{\rho_{b} g H^{2}}{2 D_{v}}
$$

where $H$ is the depth (here is $5 \mathrm{~m}$ ), $\rho_{b}$ is the initial buoyant density (subtract the fluid density $\rho_{f}$ from the initial total density $\rho$, i.e., $\rho_{b}=1200 \mathrm{~kg} / \mathrm{m}^{3}$ in our simulation). The value of $u_{\text {pre }}$ can be used as a preliminary check of the code implementation. Two different meshes are used in this problem: the fine mesh contains 14,676 triangle elements and the normal mesh contains 3,146 triangle elements. The element type is quadratic-displacement/linear-pressure element. The simulation time step is chosen to be logarithmically equally spaced between $10^{0}$ to $10^{4} \mathrm{~s}$ (401 steps). The computation time is about 1 minute on the fine mesh.

\subsection{Simulation results}

Unless otherwise stated, the results have been verified that they are not mesh-sensitive. In our simulation, $u_{\text {pre }}=$ $-0.02212059 \mathrm{~m}$ and the instantaneous/immediate undrained deformation are depicted in Figs. 4 and 5. Fig. 5 also shows deformations for these three cases at one specific time $t=400$ $\mathrm{s}$, from which a direct sense of the consolidation degree can be obtained. For instance, both the purple and green curves are 
above the orange curve, which suggests a faster consolidation rate in the basic case. Besides, the purple curve is unsymmetric due to a transversely isotropic $\boldsymbol{k}_{2}$ and the strong coupling between flow and deformation.

Figs. 6, 7, and 8 show the undrained responses on the whole computational domain. In these three figures, for the micro-fracture network continuum, we plot the excess pressure $p_{2}-\rho_{f} g D$ instead of the true pressure $p_{2}$, in order to visualize its change over time (Fig. 6). For all three figures, significant difference can be observed between the two pressure fields $p_{1}$ and $p_{2}$, both quantitatively and qualitatively. This is different from the typical behaviors of the naturally fractured reservoir at $t=0^{+}$due to the absence of pressure coupling (Zhang et al., 2021). The porous matrix pressure $p_{1}$ is much higher than the pressure $p_{2}$ of the micro-fracture network continuum, which is a basic non-equilibrium flow pattern in double porosity media (Zhang, 2020). In fact, $p_{1}$ in the vicinity of the strip load is even higher than the magnitude of that load. Thus we could conclude that if you adopt Eqs. (18) and (19) to investigate

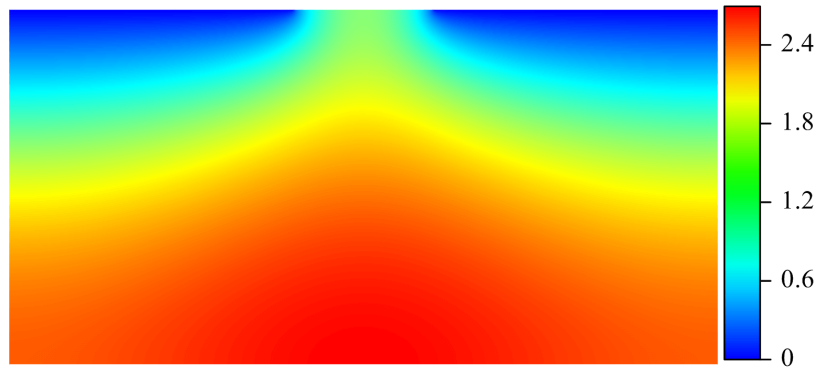

(a) Contour of $p_{2}-\rho_{f} g D$ at $1 \mathrm{~s}$ where $D$ is the depth from the top surface, the color bar is from 0 to $2.694 \mathrm{kPa}$

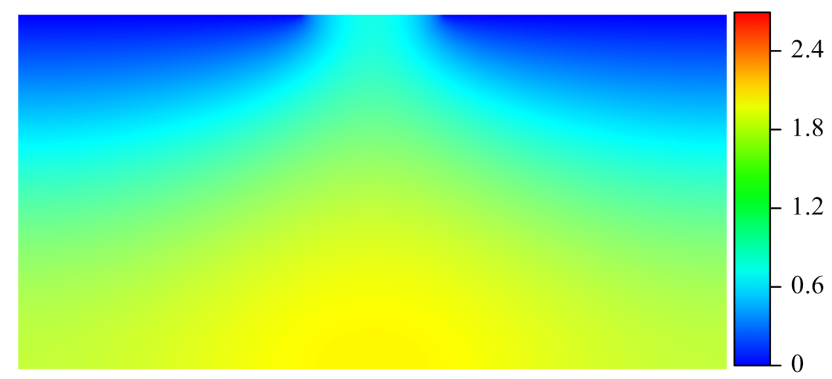

(c) Contour of $p_{2}-\rho_{f} g D$ at $100 \mathrm{~s}$ the coupled flow and deformation of double porosity media, you may never observe the stage when $p_{2}$ drops rapidly and $p_{1}$ remains unchanged. The non-equilibrium flow happens if there exists pressure difference and it promotes fluid mass transfer, thus the dissipation of porous matrix pressure $p_{1}$ is mainly through mass transfer while the contribution of flux is tiny.

Now we compare the differences among Figs. 6, 7, and 8. First, transverse isotropy in $k_{2}$ could change the pattern of $p_{2}$ immediately, which is confirmed by Fig. 7a. The contour lines are skewed and inclined. Second, in this example, since the drainage path is from the bottom to the top, the transversely isotropic $\boldsymbol{k}_{2}$ weakens the overall drainage capacity. As a result, $p_{2}$ in Fig. $7 \mathrm{a}$ is higher than that in Fig. 6a. Third, for the non-Darcy flow case, the shape of the contour line in Fig. $8 \mathrm{a}$ is exactly the same as that in Fig. 6a with scaled values. Finally, the undrained responses of the weighted pressure $\bar{p}=\alpha_{1} p_{1}+\alpha_{2} p_{2}$ are identical among three cases. Since the undrained responses of $p_{2}$ are quite different among three cases, it is fair to expect slight differences in the distribution

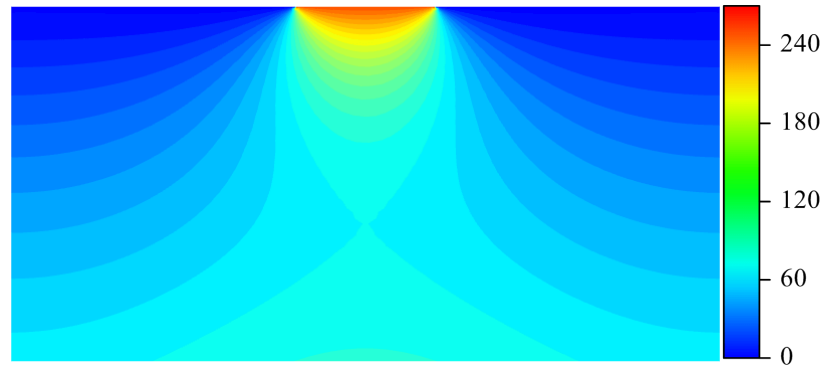

(b) Contour of $p_{1}$ at $1 \mathrm{~s}$, the color bar is from 0 to $270.1 \mathrm{kPa}$

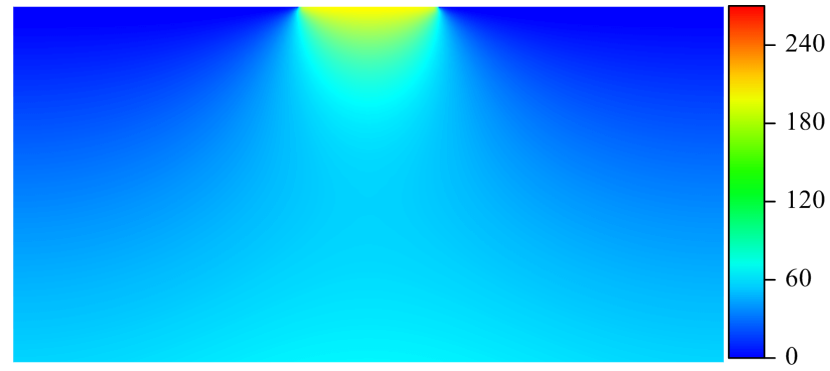

(d) Contour of $p_{1}$ at $100 \mathrm{~s}$

Fig. 6. The pressure fields when $\boldsymbol{k}_{2}$ is isotropic and the flow in the matrix continuum follows Darcy's law.

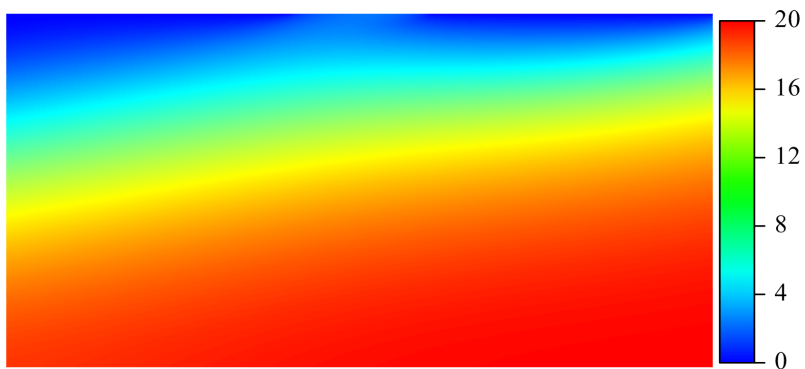

(a) Contour of $p_{2}-\rho_{f} g D$ at $1 \mathrm{~s}$, the color bar is from 0 to $20 \mathrm{kPa}$

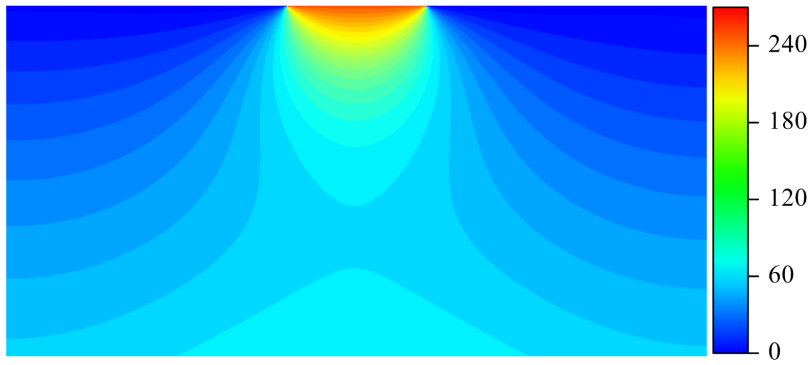

(b) Contour of $p_{1}$ at $1 \mathrm{~s}$, the color bar is from 0 to $270 \mathrm{kPa}$

Fig. 7. The pressure fields when $\boldsymbol{k}_{2}$ is transversely isotropic and the flow in the matrix continuum follows Darcy's law. 


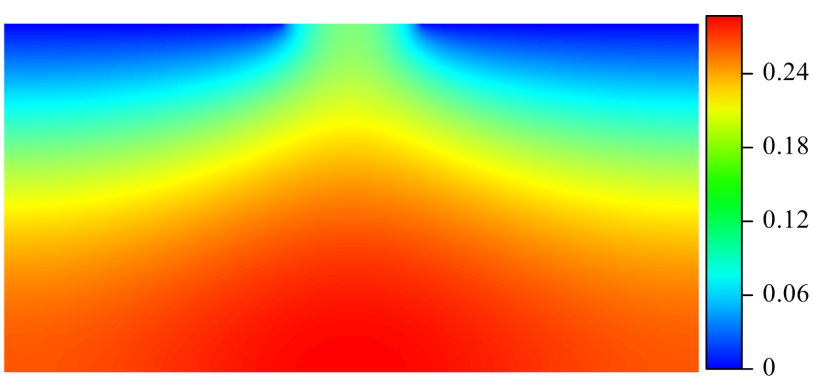

(a) Contour of $p_{2}-\rho_{f} g D$ at $1 \mathrm{~s}$, the color bar is from 0 to $0.287 \mathrm{kPa}$

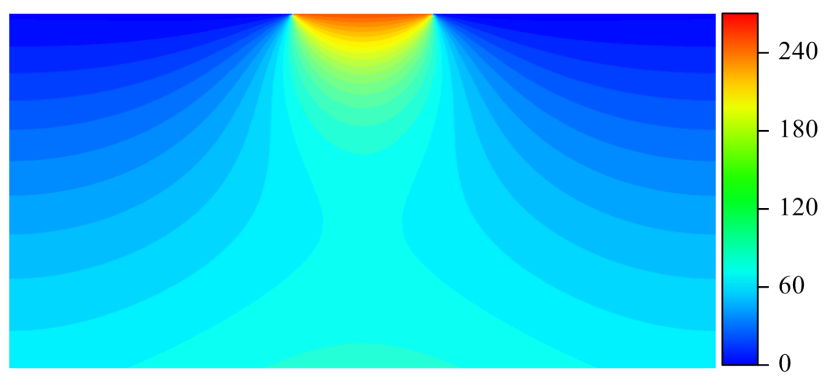

(b) Contour of $p_{1}$ at $1 \mathrm{~s}$, the color bar is from 0 to $270.3 \mathrm{kPa}$

Fig. 8. The pressure fields when $\boldsymbol{k}_{2}$ is isotropic and the flow in the matrix continuum follows non-Darcy's law.

of $p_{1}$ at $t=0^{+}$, which again matches results in Figs. 6, 7, and 8.

We are also interested in the pressure dissipations in these three cases. We plot the evolutions of weighted pressure $\bar{p}$ at one specific point (point B) as well as its average over the whole domain. The results are shown in Figs. 9 and 10, which are somehow surprising. First of all, the result of $\bar{p}$ at point $\mathrm{B}$ is mesh-sensitive in the non-Darcy case, while the meshsensitivity is not apparent when plotting the average of $\bar{p}$ over the whole domain. Second, for point B, the shapes of the red curve and the blue curve are quite different from each other. However, in Fig. 10, the red curve and the blue curve are close to each other. This characteristic in Fig. 10 is consistent with Figs. 5 and 11. Therefore, an obvious question would be why in the case of non-Darcy flow, the behavior of $\bar{p}$ at one point is so "strange"? To answer this important question, we should recall our non-Darcy flow constitutive laws. Since the contribution of flux is tiny, the only possibility would come from the leakage parameter $\gamma$. The leakage parameter $\gamma$ is a function of $\varphi_{\max }$ by using Eq. (27). Therefore, the "strange" behavior comes from the heterogeneous (non-smooth) pattern of $\varphi_{\max }$, and $\varphi_{\max }$ around point $\mathrm{B}$ is rapidly changing with time, as shown in Fig. 12. In this figure, in order to analyze the leakage parameter $\gamma$, the range of the color bar is from $\lambda_{\min } /\left(\rho_{f} g\right)=0$ to $\lambda_{\max } /\left(\rho_{f} g\right)=2$. To summarize, the nonDarcy equation of $\gamma$ could make the $p_{1}$ field not smooth and

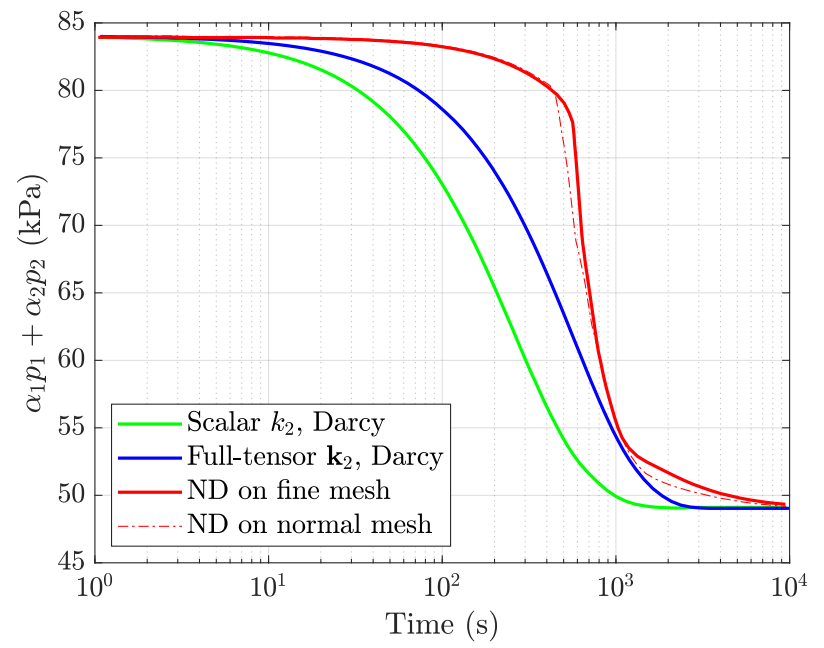

Fig. 9. Evolutions of weighted pressure $\bar{p}=\alpha_{1} p_{1}+\alpha_{2} p_{2}$ at point $\mathrm{B}(5,0)$ m. "ND" means non-Darcy equation of $\gamma$.

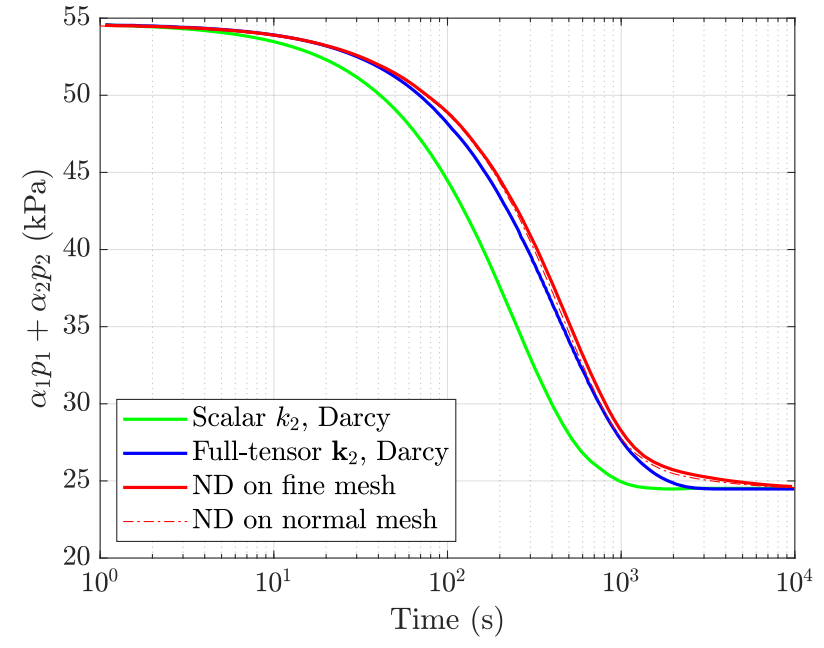

Fig. 10. Evolutions of weighted pressure $\bar{p}=\alpha_{1} p_{1}+\alpha_{2} p_{2}$ (average over the whole domain). "ND" means non-Darcy equation of $\gamma$.

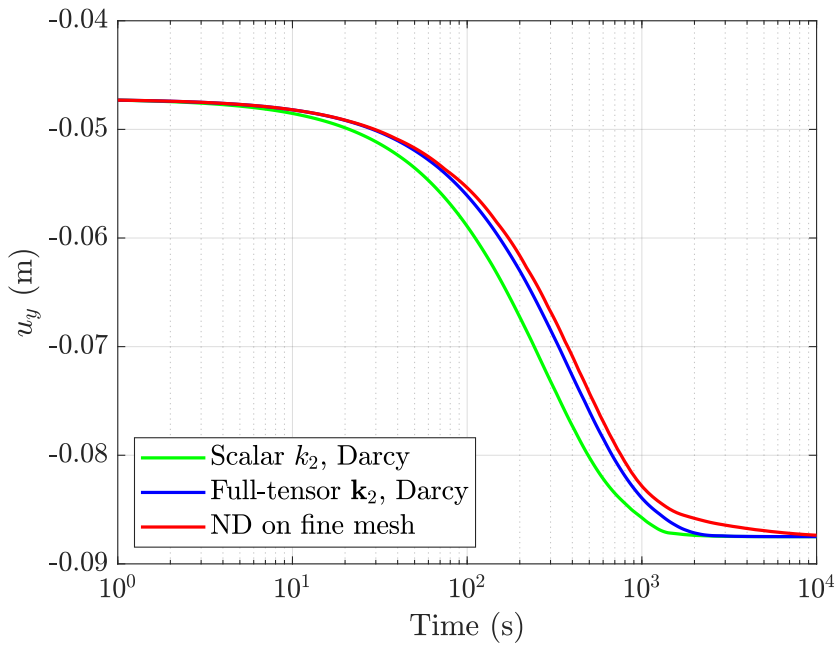

Fig. 11. Evolutions of vertical displacement $u_{y}$ at point A $(5,5) \mathrm{m}$. "ND" means non-Darcy equation of $\gamma$.

you might need an extremely fine mesh to resolve this issue (computational prohibitive at this moment). The experimental techniques are also currently not capable to verify the numerical results. Nevertheless, this mesh-sensitivity does not affect 


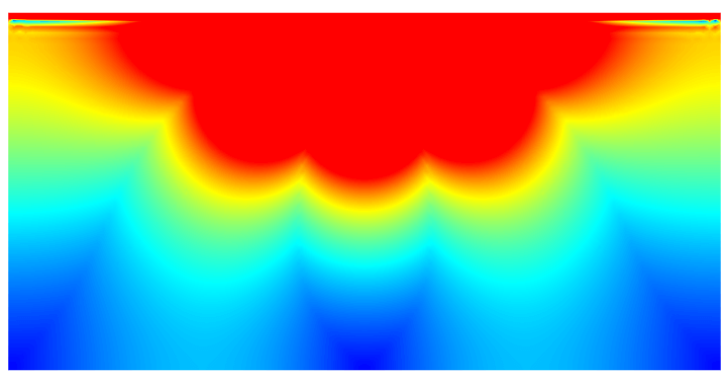

(a) $1 \mathrm{~s}$

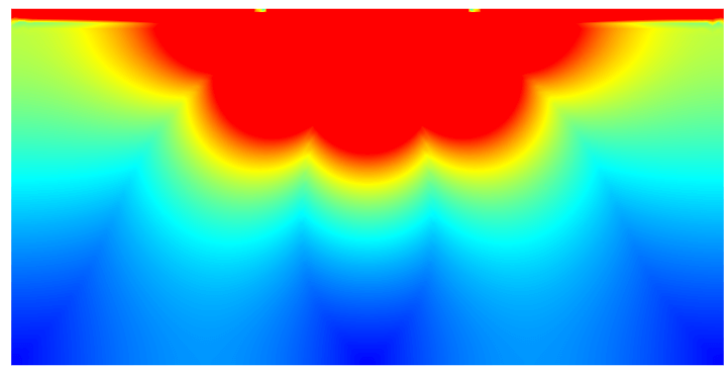

(c) $100 \mathrm{~s}$

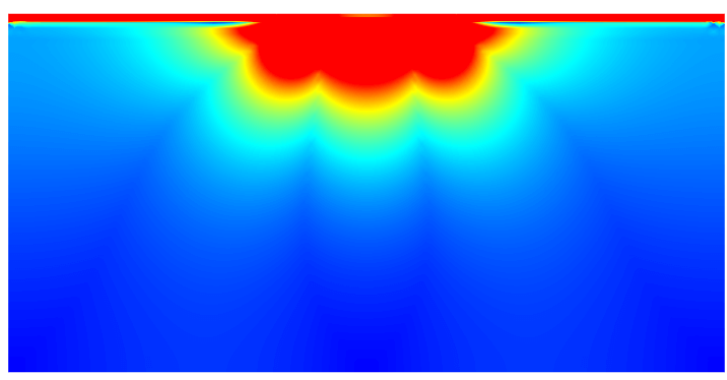

(e) $500 \mathrm{~s}$

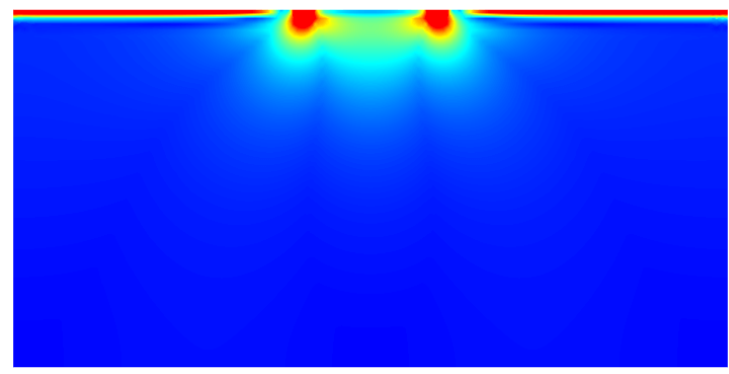

(g) $1000 \mathrm{~s}$

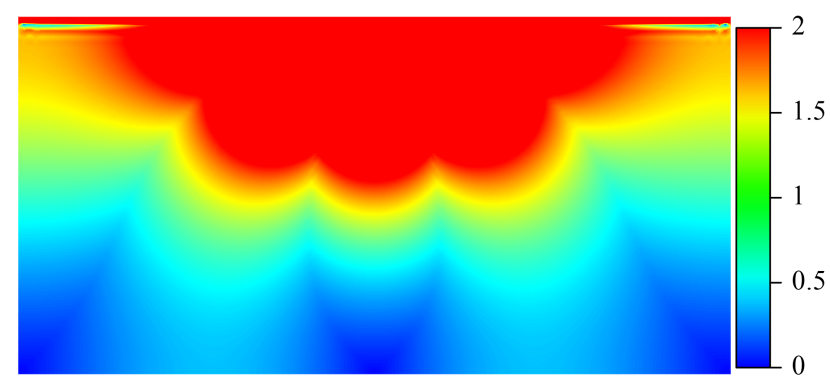

(b) $1 \mathrm{~s}$

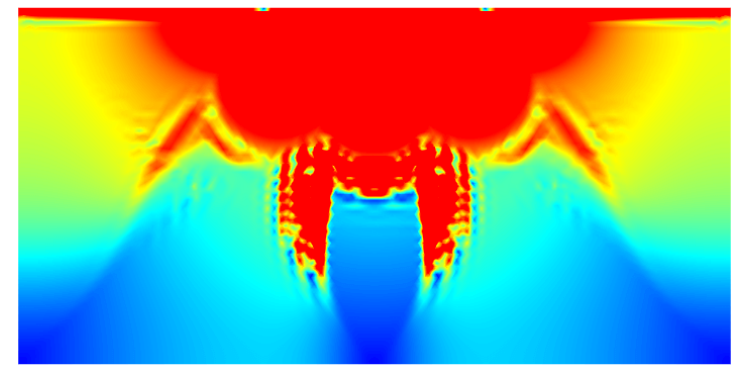

(d) $100 \mathrm{~s}$

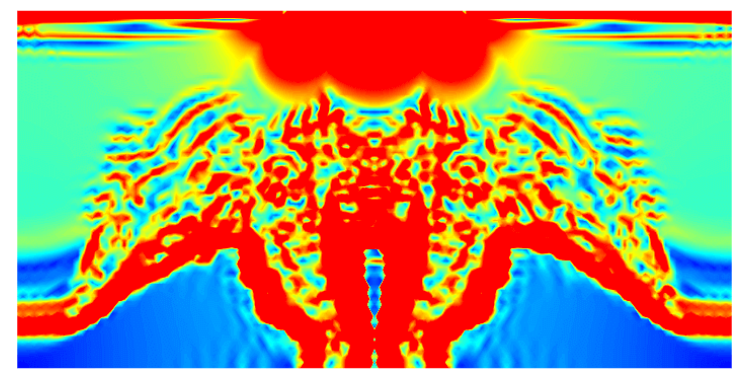

(f) $500 \mathrm{~s}$

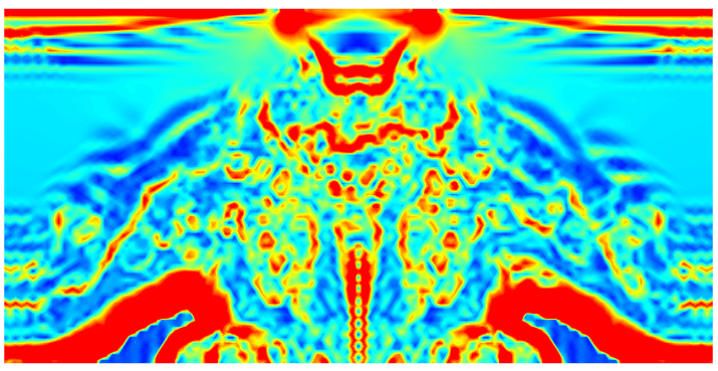

(h) $1000 \mathrm{~s}$

Fig. 12. The evolution of $\varphi_{\max } /\left(\rho_{f} g\right)$ for Darcy flow (left column) and non-Darcy flow (right column, fine mesh).

the global pressure dissipation and it is a meaningful attempt to try a dynamic $\gamma$ in double porosity modeling analogous to the multi-rate mass transfer model (Geiger et al., 2013; Tecklenburg et al., 2016).

\section{Closure}

In this work, the new treatment is the combination of transverse isotropy and non-Darcy flow. Transverse isotropy allows us to capture new excess pressure patterns in the fracture continuum. Furthermore, the dynamic leakage parameter $\gamma$ could lead to a dramatic change of potential gradient in a local area. Both the transverse isotropy and non-Darcy flow would affect the foundation settlement and global pressure dissipation behaviors. The goal of this work is to accommodate more possible features (on the constitutive level) for future geomechanics simulators and to provide some possible (theoretical) explanations for experimental findings.

\section{Acknowledgement}

The author is grateful to anonymous reviewers for their constructive comments.

Open Access This article is distributed under the terms and conditions of the Creative Commons Attribution (CC BY-NC-ND) license, which permits 
unrestricted use, distribution, and reproduction in any medium, provided the original work is properly cited.

\section{References}

Bai, M., Elsworth, D., Roegiers, J. C. Multiporosity/multipermeability approach to the simulation of naturally fractured reservoirs. Water Resources Research, 1993, 29(6): 1621-1633.

Baker, J. W., Chen, Y. Ground motion spatial correlation fitting methods and estimation uncertainty. Earthquake Engineering and Structural Dynamics, 2020, 49(15): 1662-1681.

Barenblatt, G., Zheltov, I., Kochina, I. Basic concepts in the theory of seepage of homogeneous liquids in fissured rocks. Journal of Applied Mathematics and Mechanics, 1960, 24(5): 1286-1303.

Chen, Y., Baker, J. W. Spatial correlations in cybershake physics-based ground-motion simulations. Bulletin of the Seismological Society of America, 2019, 109(6): 24472458.

Chen, Y., Baker, J. W. Community detection in spatial correlation graphs: Application to non-stationary ground motion modeling. Computers and Geosciences, 2021, 154: 104779.

Chen, Y., Baker, J. W., Bradley, B. A. Nonstationary spatial correlation in new zealand strong ground-motion data. Earthquake Engineering and Structural Dynamics, 2021, $1-20$.

Cheng, A. H. D. Poroelasticity. New York, USA, Springer, 2016.

Coussy, O. Poromechanics. Chichester, UK, John Wiley and Sons Ltd, 2004.

Cui, Y., Lu, C., Wu, M., et al. Review of exploration and production technology of natural gas hydrate. Advances in Geo-Energy Research, 2018, 2(1): 53-62.

Geiger, S., Dentz, M., Neuweiler, I. A novel multirate dualporosity model for improved simulation of fractured and multiporosity reservoirs. SPE Journal, 2013, 18(4): 670684.

Hansbo, S. Aspects of vertical drain design: Darcian or nonDarcian flow. Géotechnique, 1997, 47(5): 983-992.

Kazemi, H., Merrill, L., Porterfield, K., et al. Numerical simulation of water-oil flow in naturally fractured reservoirs. Society of Petroleum Engineers Journal, 1976, 16(6): 317-326.

Khalili, N., Valliappan, S., Wan, C. F. Consolidation of fissured clays. Géotechnique, 1999, 49(1): 75-89.

Kim, J., Sonnenthal, E. L., Rutqvist, J. Formulation and sequential numerical algorithms of coupled fluid/heat flow and geomechanics for multiple porosity materials. International Journal for Numerical Methods in Engineering, 2012, 92(5): 425-456.

Lemonnier, P., Bourbiaux, B. Simulation of naturally fractured reservoirs. State of the art: Part 2-matrix-fracture transfers and typical features of numerical studies. Oil and Gas Science and Technology-Revue de l'Institut Français du Pétrole, 2010, 65(2): 263-286.

Lewis, R. W., Schrefler, B. A. The Finite Element Method in the Static and Dynamic Deformation and Consolidation of Porous Media. Chichester, UK, John Wiley and Sons Ltd, 1998.

Li, D., Zha, W., Liu, S., et al. Pressure transient analysis of low permeability reservoir with pseudo threshold pressure gradient. Journal of Petroleum Science and Engineering, 2016, 147: 308-316.

Li, Z., Ripepi, N., Chen, C. Using pressure pulse decay experiments and a novel multiphysics shale transport model to study the role of Klinkenberg effect and effective stress on the apparent permeability of shales. Journal of Petroleum Science and Engineering, 2020, 189: 107010.

Liao, D., Yang, Z. Effect of fabric anisotropy on bearing capacity and failure mode of strip footing on sand: An anisotropic model perspective. Computers and Geotechnics, 2021, 138: 104330.

Liao, D., Yang, Z., Xu, T. $J_{2}$-deformation-type soil model coupled with state-dependent dilatancy and fabric evolution: Multiaxial formulation and FEM implementation. Computers and Geotechnics, 2021, 129: 103674.

Ranjbar, E., Hassanzadeh, H. Matrix-fracture transfer shape factor for modeling flow of a compressible fluid in dualporosity media. Advances in Water Resources, 2011, 34(5): 627-639.

Rutqvist, J., Stephansson, O. The role of hydromechanical coupling in fractured rock engineering. Hydrogeology Journal, 2003, 11: 7-40.

Sarma, P., Aziz, K. New transfer functions for simulation of naturally fractured reservoirs with dual porosity models. SPE Journal, 2006, 11(3): 328-340.

Shao, J., Zhang, Q., Sun, W., et al. Numerical simulation on non-Darcy flow in a single rock fracture domain inverted by digital images. Geofluids, 2020a, 2020: 8814327.

Shao, J., Zhang, Q., Wu, X., et al. Investigation on the water flow evolution in a filled fracture under seepage-induced erosion. Water, 2020b, 12(11): 3188.

Shao, J., Zhang, Q., Zhang, W., et al. Effects of the borehole drainage for roof aquifer on local stress in underground mining. Geomechanics and Engineering, 2021, 24(5): 479-490.

Tecklenburg, J., Neuweiler, I., Carrera, J., et al. Multi-rate mass transfer modeling of two-phase flow in highly heterogeneous fractured and porous media. Advances in Water Resources, 2016, 91: 63-77.

Villamor Lora, R., Ghazanfari, E., Asanza Izquierdo, E. Geomechanical characterization of Marcellus shale. Rock Mechanics and Rock Engineering, 2016, 49(9): 34033424.

Wang, Z., Zhang, Q., Shao, J., et al. New type of similar material for simulating the processes of water inrush from roof bed separation. ACS Omega, 2020, 5(47): 30405-30415.

Warren, J., Root, P. The behavior of naturally fractured reservoirs. Society of Petroleum Engineers Journal, 1963, 3(3): 245-255.

Xiao, S., Yue, Z. Axisymmetric BEM analysis of one-layered transversely isotropic halfspace with cavity subject to 
external loads. Engineering Analysis with Boundary Elements, 2020, 121: 91-103.

Yan, X., Huang, Z., Zhang, Q., et al. Numerical investigation of the effect of partially propped fracture closure on gas production in fractured shale reservoirs. Energies, 2020, 13(20): 5339.

Yan, X., Sun, H., Huang, Z., et al. Hierarchical modeling of hydromechanical coupling in fractured shale gas reservoirs with multiple porosity scales. Energy and Fuels, 2021, 35(7): 5758-5776.

Yang, Y., Yu, H., Yuan, Y., et al. 1 g Shaking table test of segmental tunnel in sand under near-fault motions. Tunnelling and Underground Space Technology, 2021, 115: 104080.

Ye, D., Liu, G., Gao, F., et al. A multi-field coupling model of gas flow in fractured coal seam. Advances in Geo-Energy Research, 2021, 5(1): 104-118.

Zhang, Q. Hydromechanical modeling of solid deformation and fluid flow in the transversely isotropic fissured rocks. Computers and Geotechnics, 2020, 128: 103812.

Zhang, Q., Chen, Y., Yang, Z., et al. Multi-constitutive neural network for large deformation poromechanics problem. Paper 132 Presented at Third Workshop on Machine Learning and the Physical Sciences (NeurIPS 2020), Vancouver Convention Centre, Vancouver, BC, Canada,
11 December, 2020.

Zhang, Q., Wang, Z. Spatial prediction of loose aquifer water abundance mapping based on a hybrid statistical learning approach. Earth Science Informatics, 2021, 14: 13491365.

Zhang, Q., Yan, X., Shao, J. Fluid flow through anisotropic and deformable double porosity media with ultra-low matrix permeability: A continuum framework. Journal of Petroleum Science and Engineering, 2021, 200: 108349.

Zhang, W., Mehrabian, A. Poroelastic solution for the nonlinear productivity index of wells in stress-sensitive reservoir rocks. SPE Journal, 2020, 26(1): 68-82.

Zhang, W., Mehrabian, A. Nonlinear and non-local analytical solution for Darcy-Forchheimer flow through a deformable porous inclusion within a semi-infinite elastic medium. Journal of Fluid Mechanics, 2021, 91: A49.

Zhao, L., Jiang, H., Wang, H., et al. Representation of a new physics-based non-Darcy equation for low-velocity flow in tight reservoirs. Journal of Petroleum Science and Engineering, 2020, 184: 106518.

Zhu, X., Zhang, Q., Zhang, W., et al. Experimental study on the basic properties of a green new coal mine grouting reinforcement material. ACS Omega, 2020, 5(27): 1672216732. 